\title{
Spectrum Sensing Algorithm Based on Fisher Classification
}

\author{
Wenxiang Guo a, Zhiyong Yu, Rui Jin \\ Xi'an Hong Qing High Tech Research Institute, Xi'an, China \\ aGwenxiang93@foxmail.com
}

\begin{abstract}
This paper presents a novel spectrum sensing algorithm based on Fisher classification for spectrum sensing speed, accuracy and reliability. With application of the pattern recognition theory to the process of specetrum sensing modelling, the spectral holes are employed to the greatest possible advantage and the sensing performaces are improved at low signal-noise-ratio (SNR). The signal power and SNR characterize the electromagnetic environment from the data for training. The simulation result shows that the novel algorithm has a better performance under the low SNR conditions, and the detection probability of spectrum sensing can go up in propotion to the number of the historical data.
\end{abstract}

Keywords: Pattern Recognition, Fisher Classification, Spectrum Sensing, Historial Data.

\section{Introduction}

The insufficient spectrum resources cannot be fully satisfied with the need for the modern high speed communication with the fixed spectrum allocation strategy. The dynamic spectrum allocation strategy, therefore, is proposed to improve the spectrum utilization efficiency and alleviate this contradiction. Cognitive radio was proposed originally and developed by I. Mitola, J, by J. Magure, G.Q, by J.O.Neel and so on, which solved the problem by introducing spectrum sharing mechanism in dynamic spectrum allocation strategy. As one of a very important node, the spectrum sensing has been particularly topical for the cognitive radio, which can be used to get awareness of the spectrum usage and existence of primary users in a geographical area. The core of spectrum sensing is to detect spectral holes with high speed and accuracy, so as to the secondary users' in time access or exit the licensed spectrum for the primary users.

During the past 30 years, lots of sensing methods have been proposed, each of which has its own advantages and disadvantages. Energy detection [1-3] is quite a popular algorithm because it is simple in theory, low in computational complexity and easy to implement. However, the detection threshold is difficult to determine, which is sensitive to the uncertainty of noise fluctuation. And the detection probability decreases rapidly under the low SNR conditions. Matched filter [4-5] uses principle of coherent detection to maximize the SNR of the received signal, and it converges fast and has a superior detection performance. However, the strict phase synchronization and prior information conditions make it practically infeasible. Cyclostationary feature detection [6-7] makes full use of differences between the statistical features of the signal and the noise to adapt the algorithm to lower SNR conditions. However, the complexity is high and the amount of computation is large, which results in a decrease in detection performance when it needs real time detection. In recent years, many novel algorithms have been proposed. Under the low SNR conditions, the detection probability is effectively improved with the spectrum sensing algorithm based on LMS [8], and the noise uncertainty can be avoided, however, it is limited to small sample data. Spectrum sensing based on subspace projection [9] is realized. Differences between signal subspace and noise subspace projections are compared to realize a fast detection process, but the detection reliability under low SNR conditions is also bad. For all algorithms above, none can be used to realize a satisfying spectrum sensing performance under the low SNR. So, based on linear discriminant theory, the paper applies Fisher classification to spectrum sensing technology, and the sensing performance is improved a lot under low SNR conditions.

This paper deals with spectrum sensing performance under low SNR conditions based on Fisher classification. Analyzing the sensing modeling, no doubt, it belongs to a two-group classification question, so, Fisher classification is proposed, which has a great advantage on this type of problems. The proposed algorithm makes full use of historical data to improve the intelligence of sensing 
process and improves the performances of speed, accuracy and reliability. The simulation result shows that when the historical data is large enough, the Fisher classification algorithm can guarantee a high detection probability and fast sensing speed at low SNR.

\section{Spectrum Sensing Model}

Spectrum sensing is essentially a binary assumption problem. The goal of detecting primary users is to ascertain their presence or absence. And, the model is as shown in formula (1),

$$
\begin{array}{ll}
\mathrm{H}_{0}: x(n)=u(n) & n=1,2, \cdots, N \\
\mathrm{H}_{1}: x(n)=h s(n)+u(n) & n=1,2, \cdots, N
\end{array}
$$

Where, $s(n)$ is the signal transmitted for the main user, $u(n)$ is AWGN (Additive White Gaussian Noise), $x(n)$ is a signal received from the secondary user and $h$ is the channel gain. The signal components are analyzed and compared to determine whether there is a spectral hole. $\mathrm{H} 0$ represents the absence of primary users and $\mathrm{H} 1$ stands for the presence of them.

Ideally, when the licensed band is occupied by the primary users, the result is H1, if not, it will be H0. To make a clear separation, the linear discriminant analysis method of Fisher classification is used. The noise in the actual environment always exists, and different degrees of influence on the reliability of results at different SNR, so corresponding probability values are selected to represent the influence. The differences between the actual situation and the theoretical judgment is classified as the probability of missed detection, the probability of detection and the probability of false alarming.

(1) Probability of missed detection $\mathrm{P}_{M D}$. In practice, primary users occupy the licensed frequency band, but the result is that there is a spectral hole. This is the probability of missed detection. If the value is too high, the primary users will be subject to interference. At this time, the licensed band is occupied by the primary user, but the secondary users are mistaken for the access to the licensed band, which will interrupt the normal communication of the primary users.

$$
\mathrm{P}_{\mathrm{MD}}=P\left(H_{0} / H_{1}\right)
$$

(2) Probability of detection $P_{D}$. Primary users occupy the licensed frequency band and the result is also the same. This is the detection probability. A high detection probability will not cause any interference to the primary user, and it is beneficial to quick access to the licensed frequency band from the secondary users and increases the utilization efficiency.

$$
\mathrm{P}_{\mathrm{D}}=P\left(H_{1} / H_{1}\right)
$$

(3) Probability of false alarming $\mathrm{P}_{\mathrm{FA}}$. There exist spectral holes in the licensed frequency band, the judgment results are that primary users occupy the licensed frequency band. This is the false alarming probability. A high false alarming probability will reduce the utilization efficiency, because the spectrum holes are not allocated to secondary users with efficiency, resulting in a waste of resources.

$$
\mathrm{P}_{\mathrm{FA}}=P\left(H_{1} / H_{0}\right)
$$

\section{Fisher Classification Application to Spectrum Sensing}

\subsection{Fisher Classification Analysis}

Fisher classification is a linear discriminant analysis method, which is mainly used in the field of pattern recognition and in solving the 'dimension disaster' problem [12] efficiently. The basic idea of Fisher classification is to map problems that cannot be handled in high-dimensional space to lowdimensional space for processing. Spatial projection is its basic processing method. It projects the sample data in $n$ dimensional spaces into a certain direction and converts it into one-dimensional data for processing. The projection direction determines the discrimination effect, which affects the accuracy of spectrum sensing in result. Certainly, there is always a best projection direction so that the sample data is best separated. The aim of Fisher linear discriminant analysis is to find the optimal projection direction $w$ by establishing a mathematical model and calculating it, as shown in Figure.1. 
In figure (a), the projection directions are not suitable, and the sample data cannot be distinguished after the projections. In figure (b), there is a proper projection direction, after the projection, the two types of sample data can be clearly distinguished.

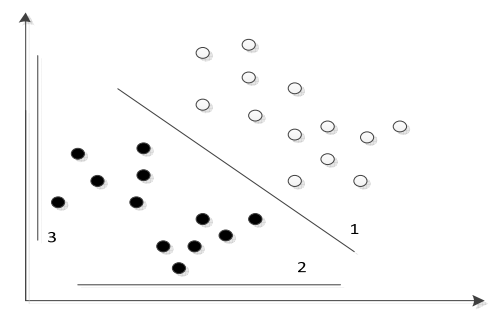

(a)

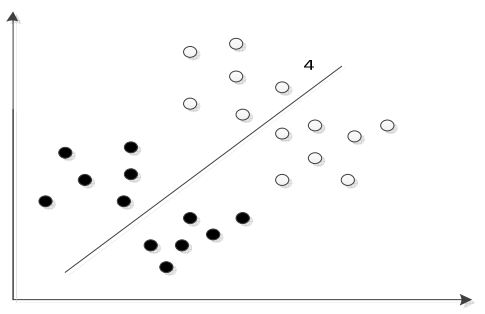

(b)

Fig.1 Fisher linear discrimination principle diagram

Assuming there is a sample set $\mathrm{X}=\left\{x_{i} \mid x_{i} \in R^{n}, i=1,2, \cdots, N\right\}$, and $x_{i}$ belongs to class $\omega_{1}$ or class $\omega_{2}$, making a projection transformation on each sample, $y_{i}=\left(w \cdot x_{i}\right)$. The projection direction determines the degree of separation after the projection of the sample data, affects the judging result of the sample data, and it needs to train the projection direction through a large amount of historical data to ensure an optimal and reliable result

\subsection{Sensing Process of Fisher Classification}

Spectrum sensing based on fisher classification is through the historical data to find the optimal projection direction, so that the two classes can be separated more clearly. Defined on the model, it shows that smaller the dispersion is within the class, better the separation performance will be. The mathematical formula derivation is characterized as

$$
\begin{array}{r}
J(w)=\frac{\overline{S_{b}}}{\overline{S_{w}}}=\frac{\left(\overline{m_{1}}-\overline{m_{2}}\right)^{2}}{\overline{S_{1}}+\overline{S_{2}^{2}}} \\
\overline{S_{i}^{2}}=\sum_{y \in \overline{w_{i}}}\left(y-\overline{m_{i}}\right)^{2}
\end{array}
$$

Where, $\overline{m_{1}}$ and $\overline{m_{2}}$ are mean values of the two class samples, $\overline{S_{1}}$ and $\overline{S_{2}}$ are the dispersion values, $\overline{S_{b}}$ and $\overline{S_{w}}$ are the inter-class dispersion degree and the intra-class dispersion degree of the historical data respectively.

Equation (6) shows that it does not include the projection direction wexteriorly, but the analysis shows that it is implicit of projection direction ${ }^{w}$, deduced as follows

$$
\begin{gathered}
\overline{m_{i}}=\frac{1}{N_{i}} \sum_{y \in \bar{w}_{i}} y=w^{T} \frac{1}{N_{i}} \sum_{x \in w_{i}} x=w^{T} m_{i} \\
\overline{S_{b}}=\left(\overline{m_{1}}-\overline{m_{2}}\right)^{2}=\left[w^{T}\left(m_{1}-m_{2}\right)\right]^{2} \\
=w^{T}\left(m_{1}-m_{2}\right)\left(m_{1}-m_{2}\right)^{T} w \\
=w^{T} S_{b} w \\
\overline{S_{i}^{2}}=\sum_{y \in \overline{w_{i}}}\left(y-\overline{m_{i}}\right)^{2}=\sum_{x \in w_{i}}\left(w^{T} x-w^{T} m_{i}\right)^{2} \\
=w^{T} \sum_{x \in w_{i}}\left(x-m_{i}\right)\left(x-m_{i}\right)^{T} w \\
=w^{T} S_{i} w \\
\overline{S_{w}}=\overline{S_{1}{ }^{2}}+\overline{S_{2}{ }^{2}}=w^{T} S_{w} w
\end{gathered}
$$

The Fisher classification criterion function is

$$
J(w)=\frac{w^{T} S_{b} w}{w^{T} S_{w} w}
$$

Equation (12) is a generalized Rayleigh quotient, which can be solved by the Lagrange multiplier method. Assuming that $w^{T} S_{w} w=k \quad(k \neq 0)$, we can get the Lagrange function

$$
L(w, \lambda)=w^{T} S_{b} w-\lambda\left(w^{T} S_{w} w-k\right)
$$


The ${ }^{w}$ partial value obtained by (13) is 0 , getting

$$
\frac{\partial L(w, \lambda)}{\partial w}=2 S_{b} w-2 \lambda S_{w} w=0
$$

Then, the best projection direction $w^{*}$ would be determined by $S_{b} w^{*}=\lambda S_{w} w^{*}$. When $S_{w}$ is a nonsingular, it will get $S_{w}^{-1} S_{b} w^{*}=\lambda w^{*}$, which is usually satisfied by spectrum data.

$$
\begin{aligned}
S_{b} w^{*} & =\left(m_{1}-m_{2}\right)\left(m_{1}-m_{2}\right)^{T} w^{*} \\
& =c\left(m_{1}-m_{2}\right)
\end{aligned}
$$

Analyzing equation (14), getting that $S_{b} w^{*}$ and $\left(m_{1}-m_{2}\right)$ correspond to the same direction, and $\mathrm{c}=\left(m_{1}-m_{2}\right)^{T} w^{*}$ is scalar. Neglecting the scale factor, we can get the best projection direction $w^{*}=S_{w}^{-1}\left(m_{1}-m_{2}\right)$.

Secondly, the threshold value is determined by $y_{0}=\frac{N_{1} \overline{m_{1}}+N_{2} \overline{m_{2}}}{N_{1}+N_{2}}$, where $N_{1}$ and $N_{1}$ are the numbers of sample data, and the final decision function is

$$
f=\operatorname{sgn}\left\{w^{*} x-y_{0}\right\}
$$

The output value $f$ is +1 or -1 , respectively reflecting whether there are spectral holes or not in the licensed band.

\section{Results Analysis}

In spectrum sensing, each false alarming will burden the subsequent data processing, and it needs to go through multiple cycles to eliminate. However, it has strong anti-leakage alarming capability. Only the continuous occurrence of missed alarming will make an impact, but the probability is extremely low. Therefore, the false alarming probability is usually strictly controlled to keep it as a constant . In the following simulation, the probability of false alarm is a small constant.

The analog channel was a Gaussian white noise channel, noise mean $u=0$, variance $\sigma_{n}=1$, and channel gain $h=1$.At each SNR, there goes the same times Monte Carlo simulations.

Detection performance between fisher classification and energy detection under different SNR are compared in fig.2. In figure.2, it shows that with the SNR increases, the detection performance of both algorithms gradually increases and eventually approaches to 1 . It is because the fact that the noise uncertainty goes down with the decrease of SNR, therefore, the detection performance of the two tend to be consistent. However, when the SNR is lower than $-2 \mathrm{~dB}$, fisher classficaton detection probability is significantly higher than that of energy detection, and it converges faster.

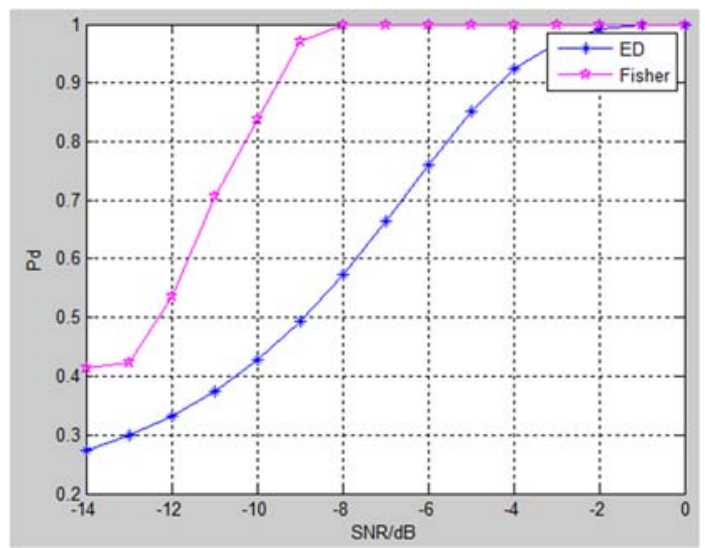

Fig.2 Fisher and ED detection probability comparison
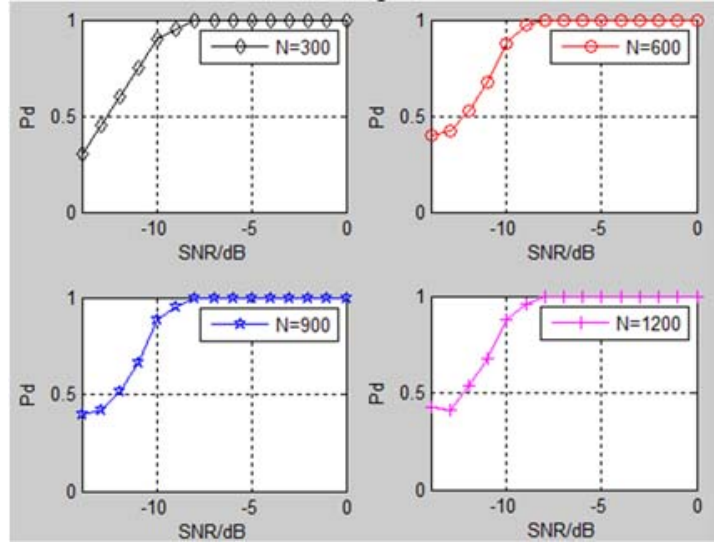

Fig.3 Detection performance with training samples curve 
In Fig.3, four simulation results are shown, which is different on historical data number. It was observed that, as the number increases, the whole detection probability under low SNR increases. With the SNR increases, the detection probability tends to 1 . However, it is not difficult to find that there may be pits in a single detection probability curve, indicating that the detection performance does not increase strictly with the increase of SNR. When the number of historical sample data is small, the detection probability at a certain SNR may decrease.

In order to show the fisher classification performance rmore clearly, a table is listed. As shown in Table 1, the columns and rows can clearly verify the conclusions drawn in Fig. 4. Although there is a case where training samples increased, the detection probability under a single SNR value decreased, but the whole trend increases, which explains the necessity of a large number of training samples.

Tab1.Detection probability on differrnt SNR and training samples

\begin{tabular}{|c|c|c|c|c|c|c|c|c|c|}
\hline$\frac{\mathrm{SNR}}{\mathrm{N}}$ & -14 & -13 & -12 & -11 & -10 & -9 & -8 & ... & 0 \\
\hline 300 & 0.30 & 0.45 & 0.60 & 0.75 & 0.90 & 0.95 & 1.00 & .. & 1.00 \\
\hline 600 & 0.40 & 0.43 & 0.53 & 0.68 & 0.88 & 0.98 & 1.00 & $\ldots$ & 1.00 \\
\hline 900 & 0.40 & 0.42 & 0.52 & 0.68 & 0.88 & 0.95 & 1.00 & $\ldots$ & 1.00 \\
\hline 1200 & 0.42 & 0.41 & 0.54 & 0.68 & 0.85 & 0.96 & 1.00 &.. & 1.00 \\
\hline
\end{tabular}

\section{Conclusion}

Fisher theory has a natural advantage on classification. This paper proposes applying fisher classification to spectrum sensing technology, ensuring a good spectrum sensing performance for speed, accuracy and reliability under low SNR conditions. And simulation results show that when the number of historical sample data is large enough, it can overcome the uncertainty of noise fluctuation and the detection probability of spectrum sensing will go up with the rise number of ahistorical data, proving the consistence between the theoretical derivation and simulation results.

\section{References}

[1]. Mohamed Shalaby, Mona Shokair and Yaser S.E.Abso. Simulation of Cognitive Radio System Applying Different.

[2]. Wireless Channel Models[J]. International Journal of Computer Networks \& Communications. Vol.5, No.2,March ,2013.

[3]. S.Taruna,Bhumika Pahwa. Simulation of Cognitive Radio Using Periodogram[J]. International Joural of Engineering Research \& Technology. Vol.2 Issue 9,September 2013.

[4]. JIN Yan-jun,ZHU Qi and ZHENG Baoyu.Optimation of dynamic Double-threshold Cooperative Spectrum Sensing Performance in Cognitive Radio Networks[J].JOURNAL OF SIGNAL PROCESSING. Vol.31,No.3,March 2015.

[5]. Gholamipour A H,Gorcin A,Celebi H,e-tal.Reconfigurable Filter Implementation of a Matchfilter Based Spectrum sensor for Cognitive Radio Systems[C]. International Symposium on Circuits and Systems ,2011:2045-2460.

[6]. ZHANG Xin-ru.Matched Filter Based Spectrum and Power Level Recognition Algorithm[D]. Chongqing University of Posts and Telecommunications,2015.

[7]. YANG Jie. Research on CyclostationarySignature Detection Algorithm of Cognitive Radio[D]. HangZhou: ZhejiangUniversity.

[8]. ZHANG Haoye, BAO Zhihua. Spectru-m Sensing Based on Cyclostationary Characteristics[J]. Communications Te-chnology, VOL,43,No.09,2010. 
[9]. WANG Fan, LU Guangyue. Spectrum Sensing Algorithm Using LMS[J].JOURNAL OF SIGNAL PROCESSING， 2016,32 (05):543-548.

[10]. SUN Yu, LU Guangyue. The Research of Spectrum Sensing Method Baesd on Subspace Projection[J]. JOURNAL OF SIGNAL PROCESSING, 2015,31(04):483-489.

[11]. ZHANG Xuegong. Pattern Recognition[M]. Beijing: Tsinghua University Press, 2010. 